\title{
'Ulcer-like projection' in uncomplicated acute type B intramural haematoma: might we prevent or protect from an unexpected event?
}

\author{
Gabriele Piffaretti $\mathbb{D}^{\mathrm{a}, *}$ and Stefano Bonardelli ${ }^{\mathrm{b}}$ \\ a Vascular-Department of Medicine and Surgery, University of Insubria School of Medicine, Varese, Italy \\ ${ }^{b}$ Vascular Surgery-Department of Surgical and Clinical Sciences, University of Brescia School of Medicine, Brescia, Italy \\ * Corresponding author. Vascular Surgery-Department of Medicine and Surgery, University of Insubria School of Medicine, via Guicciardini 9,21100 Varese, Italy. \\ Tel: +39-0332-393-259; e-mail: gabriele.piffaretti@uninsubria.it (G. Piffaretti).
}

Keywords: Type B intramural haematoma - Ulcer-like projection • Aortic adverse events

'If you do not expect the unexpected you will not find it'. (Heraclitus)

In their article entitled, 'Risk stratification of ulcer-like projection in uncomplicated acute type B aortic intramural haematoma', Chen et al. [1] report their experience about the prediction power of aortic adverse event (AAE) that some kind of ulcer-like projection (ULP) morphology might have when developed in uncomplicated acute type B intramural haematoma (B-IMH).

Despite a constant, if not growing interest surrounding $\mathrm{B}-\mathrm{IMH}$ and the perception that the presence or development of ULP might frequently lead to life-threatening $A A E$, there are still substantial equipoise in the literature regarding the type of treatment algorithm [2]. The study of Chen et al. [1] underlines once again how $\mathrm{B}-\mathrm{IMH}$ is a highly dynamic process, even if their study does not consider that also in asymptomatic patients the most dangerous period for ULP development and/or AAE after the clinical onset of B-IHM is within the first 2 weeks (e.g. the authors repeat an early computed tomography scan only if clinical changes occur). At any rate, progression towards AAE was observed in up to $40 \%$ of the patients, especially in those presenting or developing ULP which, therefore, may play a significant role in determining which type of treatment should be applied in these circumstances [3]. Furthermore, at least one-third of the observed $\mathrm{B}$-IHM was lost during follow-up, thus we can presume that this high rate of adverse progression of ULP might be even higher. Baseline data meta-analysis showed patients with complicating features of ULP were more likely to be managed with thoracic endovascular aortic repair (TEVAR), and when selective TEVAR was used in B-IMH with ULP progression, it was found to be associated with lower risk of dissection and lower risk of rupture during follow-up [4].

Given the unpredictable, rapidly changing behaviour of this treacherous type of aortic lesion, there are 2 important aspects that will may help to refine the best management strategy of $\mathrm{B}$-IMH with ULP $[5,6]$. First, identifying morphological variables unquestionably associated with progression towards AAE. What this clinical experience of Chen et al. [1] is adding new is that they were able to identify more specific morphologic criteria of such ULP that predicted AAE with high accuracy, especially the depth $\geq 5.0 \mathrm{~mm}$ of the 'crater-like' protrusion within the IMH when located in the proximal aortic segments. Secondly, identifying the best time window to repair the aortic lesion; indeed, potentially this will be the most difficult aspect to be optimized. Avoiding aorta-related mortality (ARM) due to rupture or aortic disease progression is the mainstay of the each type of treatment approach, not only in the acute setting but also during the follow-up $[7,8]$. In our personal experience, nearly $70 \%$ of the detected ULPs developed/progressed significantly within 30 days from admission. Given the fact that selective TEVAR has proven effective with a $92 \%$ freedom from ARM at 5 years, a selective but proactive early approach with TEVAR may be beneficial at preventing both AAE and ARM [6]. That said, TEVAR for B-IMH is not coming without costs: a major concern is the potential risk of endograft-induced new intimal lesion. However, this occurred rarely was not exclusively determined by the underlying aortic disease, rather a combination of factors, and most have been amenable of redo-TEVAR without additional ARM.

In conclusion, the study of Chen et al. [1] is significant because underlines that clinical and morphological details really may help operators in surgical decision-making: considering that ULP within $\mathrm{B}-\mathrm{IMH}$ is a rapidly evolving entity, and high-risk ULP is a marker of AAE despite optimal medical therapy, a more proactive operative treatment with TEVAR may be warranted in these clinical scenarios.

\section{REFERENCES}

[1] Chen L, Yang F, Liu J, Luo S, Yuan H, Fan R et al. Risk stratification of ulcer-like projection in uncomplicated acute type B aortic intramural hematoma. Eur J Cardiothorac Surg 2021;60:1032-40. 
[2] Mussa FF, Horton JD, Moridzadeh R, Nicholson J, Trimarchi S, Eagle KA Acute aortic dissection and intramural hematoma: a systematic review. JAMA 2016;316:754-63.

[3] Miyahara S, Mukohara N, Fukuzumi M, Morimoto N, Murakami $H_{\text {, }}$ Nakagiri $\mathrm{K}$ et al. Long-term follow-up of acute type $\mathrm{B}$ aortic dissection: ulcer-like projections in thrombosed false lumen play a role in late aortic events. J Thorac Cardiovasc Surg 2011;142:e25-31.

[4] Chakos A, Twindyawardhani T, Evangelista A, Maldonado G, Piffaretti G, Yan TD et al. Endovascular versus medical management of type $B$ intramural hematoma: a meta-analysis. Ann Cardiothorac Surg 2019;8: 447-55.

[5] Park GM, Ahn JM, Kim DH, Kang JW, Song JM, Kang DH et al. Distal aortic intramural hematoma: clinical importance of focal contrast enhancement on CT images. Radiology 2011;259: 100-8.

[6] Piffaretti G, Lomazzi C, Benedetto F, Pipitò N, Castelli P, Trimarchi S et al. Best medical treatment and selective stent-graft repair for acute type $B$ aortic intramural hematoma. Semin Thorac Cardiovasc Surg 2018;30: 279-87.

[7] Lavingia KS, Ahanchi SS, Redlinger RE, Udgiri NR, Panneton JM. Aortic remodeling after thoracic endovascular aortic repair for intramural hematoma. J Vasc Surg 2014;60:929-35

[8] Sueyoshi E, Nagayama H, Hashizume K, Eishi K, Sakamoto I, Uetani M. Computed tomography evaluation of aortic remodeling after endovascular treatment for complicated ulcer-like projection in patients with type B aortic intramural hematoma. J Vasc Surg 2014;59:693-9. 